\section{Clinical case reports using a novel calcium-based cement}

\author{
I. K. Bachoo, ${ }^{* 1}$ D. Seymour ${ }^{2}$ and P. Brunton ${ }^{3}$
}

VERIFIABLE CPD PAPER

IN BRIEF
Introduces a novel calcium-based
cement reported to encourage repair and
regeneration of lost dental tissue.
- Presents two case reports using this
cement for surgical endodontic purposes,
identifying its advantages over currently
used alternatives.
- Highlights the clinical handling properties
of the novel cement.
Discusses the value and future of the
material in general dental practice.

A novel calcium-based cement was launched in 2009 claiming to be a revolutionary material capable of offering a bioactive and biocompatible replacement for dentine. The calcium-based cement has been reported to encourage the repair and regeneration of lost dental tissue, preserve pulp vitality, promote pulp healing and provide a bioactive substitute for natural dentine. This article looks at two clinical cases using this new material for endodontic purposes.

\section{INTRODUCTION}

In 2009 a new product was launched claiming to be a revolutionary material capable of offering a bioactive and biocompatible replacement for dentine. The calcium-based cement is reported to preserve pulp vitality, promote pulp healing and provide a bioactive substitute for natural dentine. Its clinical indications are extensive (Table 1) being described as a restorative material suitable for use wherever dentine replacement is required. ${ }^{1}$ The reported clinical advantages of this material include quick setting time, rapid development of mechanical strength, easy handling and minimal requirement for cavity preparation. ${ }^{2,3,4}$ In a previous article $^{4}$ the physical, chemical, mechanical and biological properties of the material were discussed, including the preliminary results of in vitro and in vivo trials with this material. It is the purpose of this article to look at two clinical cases using this new material for endodontic purposes.

The endodontic indications of the novel material are similar to mineral trioxide aggregate (MTA), but are reported to offer several advantages including: ${ }^{6}$

"Former Senior House Officer in Restorative Dentistry, ${ }^{2}$ Speciality Registrar in Restorative Dentistry, ${ }^{3}$ Professor and Honourary Consultant in Restorative Dentistry, Leeds Dental Institute

${ }^{*}$ Correspondence to: Ishpinder Kaur Bachoo Email: ishpinder.bachoo@gmail.com

\section{Refereed Paper}

Accepted 19 July 2012

DOI: 10.1038/sj.bdj.2013.53

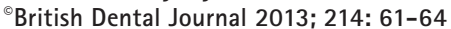

- Better consistency suited to clinical use compared to MTA

- Improved handling as a result of presentation of material

- Quicker setting time which means it eliminates two step obturation, as with MTA, and reduces the risk of bacterial contamination.

These clinical advantages over MTA would make complicated endodontic procedures more straightforward and efficient, which could translate into improved clinical results.

\section{CLINICAL CASE STUDY ONE}

\section{Presenting complaint}

Patient A, a 54-year-old male referred by their general dental practitioner following an iatrogenic distal perforation of the lower left second premolar (35) when trying to access the root canal. The patient was suffering from continuous low grade pain from the 35 which increased in severity immediately following the failed access procedure.

\section{Medical history and clinical examination}

Patient A had no medical history of note. He was a non-smoker. Extra-oral examination revealed nothing of note. Intra-oral examination revealed the 35 had a mesial-occlusal (MO) amalgam present that had been partially removed during the access procedure. The tooth
Table 1 Clinical indications of Biodentine ${ }^{T M}$

Clinical indications of Biodentine ${ }^{\mathrm{TM}}$

Temporary enamel replacement

Permanent dentine replacement

Restoration of deep and/or large coronal carious lesions (sandwich technique)

Restoration of deep cervical and/or radicular lesions

Pulp capping

Pulpotomy

Repair of root perforation

Repair of furcation perforation

Repair of perforating internal resorptions

Repair of external resorptions

Apexification

Root end filling in endodontic retrograde surgery

was coronally restorable and served as a functional tooth in both occlusion and as an abutment for a removable partial denture. There were no clinical signs of endodontic infection.

\section{Radiographic examination}

Radiographic examination (of the referring clinician's radiograph) showed a peri-apical radiograph that was poorly paralleled (Fig. 1). However, enough clinical information was present to see an endodontic file showing a perforation distally. A periapical radiolucency was also present on the 35. 


\section{Diagnosis and treatment planning}

A diagnosis of chronic periapical periodontitis and distal iatrogenic perforation 35 was made. Treatment options for the 35 were discussed at length with the patient and the decision was made to restore the perforation and endodontically treat the 35. Following this a composite core would be placed followed by a metal ceramic crown.

\section{Clinical procedure}

\section{Anaesthesia and rubber dam}

Anaesthesia was achieved via a left hand side inferior dental nerve block and buccal infiltration adjacent to the 35 . The MO amalgam in the 35 was removed under rubber dam.

\section{Surgical endodontic perforation}

The canal was identified, irrigated, dried with paper points and temporarily isolated with a cotton pledglet. The perforation was then directly observed under microscopic magnification. The distal perforation was larger than expected, measuring approximately $5 \mathrm{~mm}$ in height and $2 \mathrm{~mm}$ in width, and extended below the alveolar crest. Chemical irrigation of the perforation was performed and haemostasis achieved.

\section{Correction of the perforation and root canal treatment}

Biodentine $^{\mathrm{TM}}$, packed with an amalgam plugger, was used to restore the defect. Routine endodontic treatment was then performed once the Biodentine ${ }^{\mathrm{TM}}$ had fully set.

\section{Coronal Restoration}

Coronally, the 35 was restored with a composite core and definitively with a metalceramic crown.

\section{Review}

The post-operative radiograph (Fig. 2), prior to crown preparation, shows a very well adapted perforation repair. The area has been marked on the radiograph as it is difficult to visualise when using conventional radiographic films. The patient is completely pain free and the 35 is a functional tooth. The patient will have his six month endodontic review at the next appointment.

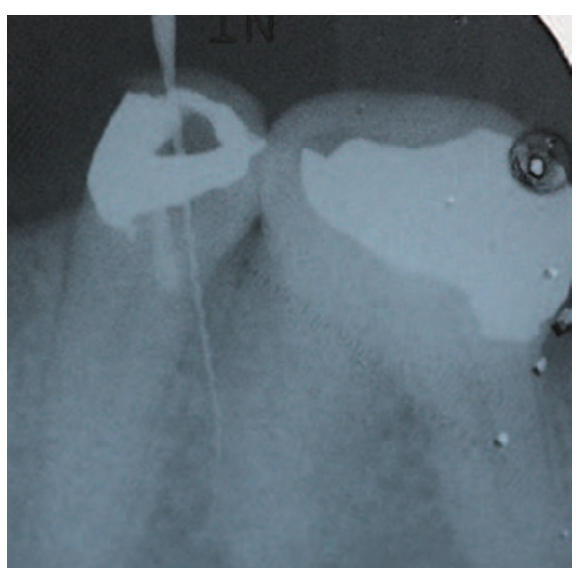

Fig 1 Pre-op long cone periapical of patient A. Note poor angulation of the peri-apical radiograph and distal perforation

\section{CLINICAL CASE STUDY TWO}

\section{Presenting complaint}

At the time of presentation, patient $\mathrm{B}$, a 47-year-old female, reported recurrent gingival swellings and a continuous dull ache associated with the upper right central incisor (11) over the past three years. There was tenderness to buccal palpation over the apex (Fig. 3). These symptoms had been ongoing following root canal treatment of the tooth.

\section{Medical history and clinical examination}

The patient is medically fit and well. She is a non-smoker and drinks alcohol on an occasional basis. The patient is a regular dental attendee and is dentally motivated. Extra-orally and tra-orally there were nil findings of relevance other than tenderness to buccal palpation.

\section{Radiographic examination}

Radiographic examination with a long cone periapical revealed a large periapical radiolucency associated with the upper right central incisor (Fig. 4).

\section{Diagnosis and treatment planning}

A diagnosis of chronic apical abscess was given for the 11. There was reason to believe that proper aseptic techniques may not have been followed at the time of the original root canal filling, and it was assumed possible that conventional orthograde re-root treatment may resolve the periapical infection as an initial measure. Throughout the conventional orthograde re-root canal treatment, however, there

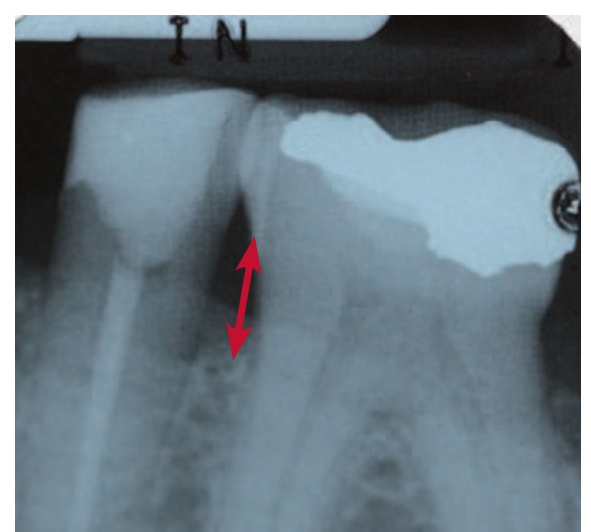

Fig 2 Post-op long cone periapical of patient A's lower left premolar. Note slightly different contrast of the Biodentine ${ }^{\mathrm{TM}}$, but generally it appears radiolucent

was continuous suppuration from the apex and an adequate apical seal could not be guaranteed. It was deemed necessary to therefore provide root resection and retrograde root filling for this tooth.

\section{Clinical procedure}

\section{Anaesthesia}

Anaesthesia was achieved through topical anaesthesia followed by high buccal and palatal infiltrations adjacent to 11 .

\section{Flap design}

A 3-sided full thickness mucoperiosteal flap was raised to perform apical surgery on the 11 .

\section{Access and root resection}

Access was gained to the surgical apical site through use of a straight handpiece and round bur, and enlarged with a fissure bur. Granulation tissue was removed at the site, followed by root end resection with the fissure bur of 2-3 $\mathrm{mm}$.

\section{Retrograde preparation} and obturation of canal

The canal was observed and approximately $3 \mathrm{~mm}$ of gutta percha removed with an ultrasonic tip. Haemostasis was achieved and the canal dried with paper points. The retrograde cavity was then obturated with Biodentine $^{\mathrm{TM}}$ and packed using an amalgam plugger. The excess cement was removed and the root end cleaned.

\section{Flap replacement}

The flap was replaced and secured with interrupted sutures. Post-operative 


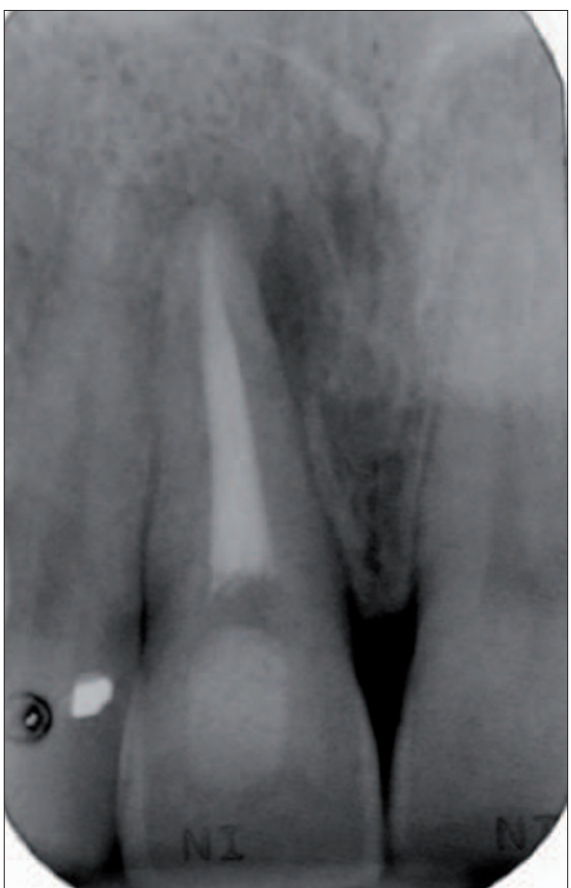

Fig 3 Pre-op view of patient B's 11, exhibiting a periapical radiolucency consistent with chronic apical periodontitis

instructions were given and a post-operative radiograph taken (Fig. 4).

\section{Review}

The tooth was reviewed one week later for suture removal and will be reviewed six months later. At one week, the surgical site was healing well with no signs of a continuing periapical pathology, and resolution of the patient's symptoms.

\section{CLINICAL OBSERVATIONS OF NOVEL MATERIAL}

\section{Manipulation and handling}

The authors found the novel material easy to manipulate and handle within the intraoral environment. In particular, handling within the surgical sites was comparable to MTA. The consistency of the material was such that packing into the root end was straightforward.

\section{Setting time}

Following mixing, the material has an adequate working time to allow for placement and plugging of the retrograde root filling. Development of the final set appeared to occur after approximately 12 minutes.

\section{Radiographic appearance}

The radiographic appearance of the material is important in order to assess for an

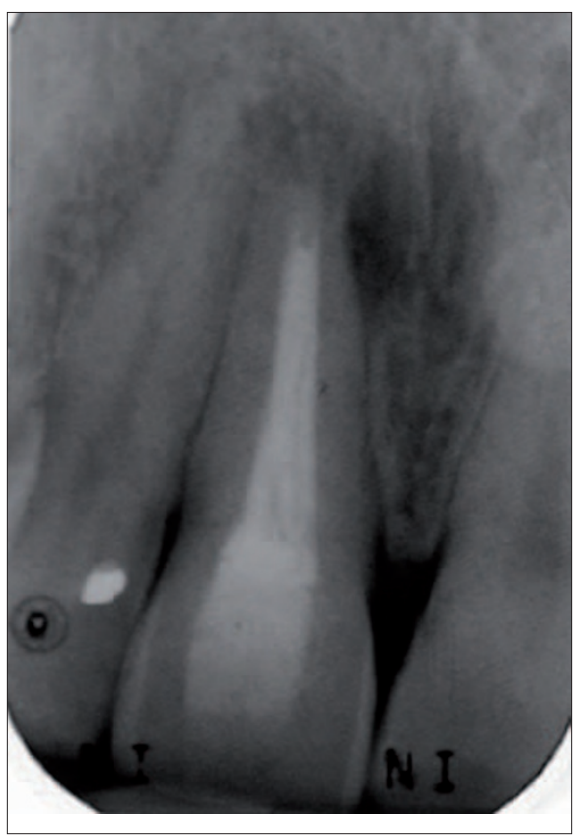

Fig 4 Post op long cone periapical of patient B's 11 following root resection and retrograde root filling with Biodentine ${ }^{T M}$. Appears to show good adaptation of material and a generally radiolucent appearance

apical seal, or for recurrent caries in cases where the material is used coronally. It is reported the material is radio-opaque as a result of the addition of zirconium dioxide. ${ }^{1}$ However, the post-op radiographs for patients A and B (Figs 2 and 4) show a faintly radiolucent appearance of the material which makes radiographic assessment of success difficult and an issue for short- and long-term follow up. ISO standard 6876:2012 governs the specific requirements for root canal sealing materials used via an orthograde approach. ${ }^{7}$ It determines that root canal sealing materials should have a minimum radiopacity equivalent to $3.0 \mathrm{~mm}$ of aluminium. The manufacturers claim that the new material, with a radiopacity equivalent of $3.5 \mathrm{~mm}$ of aluminium, satisfactorily complies with this international standard. It would appear from these two cases, however, that compliance does not necessarily translate into an ideal clinical result when this material is used for retrograde endodontics. Unfortunately there is as yet no international standard for the minimum radiopacity for retrograde filling materials.

In a related study by Filho et al. (2006), ${ }^{8}$ where a variety of root canal sealing materials were tested for their radiopacity in a retrograde capacity, it was shown a resin-based cement and zinc-oxide eugenol obtained the best radiopacity equivalent of $8.9 \mathrm{~mm}$ of aluminium. In the same study Pro Root ${ }^{\mathrm{TM}}$ MTA, another calcium-based cement similar in composition to Biodentine ${ }^{\mathrm{TM}}$, gave the lowest figure of $2.5 \mathrm{~mm}$. It appears, therefore, that alternative root sealing materials such as resin-based sealants or zincoxide eugenol may offer superior radiopacity to calcium-based cements such as Biodentine ${ }^{\mathrm{TM}}$. Clearly, further testing of Biodentine ${ }^{\mathrm{TM}}$ in retrograde and orthograde conditions, together with alternative materials, is needed to assess its comparative radiographic performance.

\section{Biocompatibility and bioactivity}

For both cases treated with Biodentine ${ }^{\mathrm{TM}}$, there were no reported sensitivity type reactions or rejection of the material. As follow up of these patients is still ongoing and without histological analysis of the tissues in contact with the material, the purported biocompatible and bioactive properties of the material cannot be confirmed. Likewise without histological analysis of the teeth treated with this new material, it is difficult to assess whether there is evidence of its reported bioactivity.

\section{Longevity}

Through post-operative follow-up it is hoped the apical seal will be able to be reviewed radiographically to assess the material's longevity

\section{CONCLUSION}

The reported biocompatible and bioactive properties of this new material combined with its mechanical properties offers an attractive solution to a number of clinical scenarios, which may occur from anywhere in the crown down to the root apex. The case reports described have further illustrated the material to be clinically practical and user-friendly. 'However, the results of on-going clinical in vivo investigations, randomised control trials, case reports and further independent clinical trials with extended follow-up periods. So far the manufacturer's claims have largely been supported by in vitro investigations and if this can transfer into clinical practice the promise of a new generation of regenerative restorative material may be realised. 
The authors would like to state that there is no affiliation between themselves and with the manufacturer (Septodont, Saint Maur Des Fossés, France) of this product.

1. Biodentine $\mathrm{T}^{\mathrm{TM}}$; Active Biosilicate Technology $\mathrm{y}^{\mathrm{TM}}$. Product Information Leaflet 2009. Septodont, Saint Maur Des Fossés, France.

2. Pradelle-Plasse N, Tran XV, Colon P. Physicochemical properties. In Goldberg M (ed) Working group of ORE-FDI, Biocompatibility or cytotoxic effects of dental composites. Oxfordshire: Coxmoor Publishing Company, 2009
3. Laurent P, Aubut V, About I. Biological properties; development of a bioactive $\mathrm{Ca}_{3} \mathrm{SiO}_{5}$-based posterio restorative material (Biodentine ${ }^{\mathrm{TM}}$ ). In Goldberg $\mathrm{M}$ (ed) Working group of ORE-FDI, Biocompatibility or cytotoxic effects of dental composites. Oxfordshire: Coxmoor Publishing Company, 2009.

4. Laurent P, Camps J, De Méo M, Déjou J, About I. Induction of specific cell responses to a $\mathrm{Ca}_{3} \mathrm{SiO}_{5}$ based posterior restorative material. Dent Mater 2008; 24: 1486-1494.

5. Bachoo I K, Seymour D, Brunton P. A biocompatible and bioactive replacement for dentine: is this a reality? The properties and uses of a novel calcium-based cement. Br Dent J 2013; 213: E5.

6. Research and Development Department Septodont, Biodentine ${ }^{T M}$; Active Biosilicate Technology ${ }^{T M}$. Internal data; scientific file, 2009

7. International Organization for Standardization. ISO standard 6876:2012. ISO, 2012. Online information available at http://www.iso.org/iso/ catalogue_detail.htm?csnumber $=45117$ (accessed November 2012)

8. Filho M T, Laitano S C, Gonçalves M, Tanomaru $J$ M G. Evaluation of the radiopacity of root-end filling materials by digitization of radiographic images. Braz J Oral Sci 2006; 5: 1018-1021. 